\title{
Simultaneous Measurement of Isotope-Free Tracer Diffusion Coefficients and Interdiffusion Coefficients in the Cu-Ni System
}

\author{
Esin A. Schulz ${ }^{1}$ Abhishek Mehta ${ }^{1}$ Irina V. Belova ${ }^{2}$ - Graeme E. Murch ${ }^{2}$. \\ Yongho Sohn ${ }^{1}$
}

Submitted: 9 May 2018/in revised form: 21 August 2018/Published online: 28 September 2018

(c) ASM International 2018

\begin{abstract}
A new formalism recently developed by Belova et al., based on linear response theory combined with the Boltzmann-Matano method, allows determination of tracer and interdiffusion coefficients simultaneously from a single, isotope-free, traditional diffusion couple experiment. An experimental methodology with an analytical approach based on the new formalism has been carried out using the model $\mathrm{Cu}-\mathrm{Ni}$ system to effectively determine tracer diffusion coefficients from an isotope-free diffusion couple experiment. $\mathrm{Cu}$ thin films were deposited in between several binary diffusion couples with varying terminal alloy compositions $(\mathrm{Cu}-25 \mathrm{Ni}, \mathrm{Cu}-50 \mathrm{Ni}, \mathrm{Cu}-75 \mathrm{Ni}, \mathrm{Ni})$. Diffusion couples were annealed at 800,900 and $1000{ }^{\circ} \mathrm{C}$, and the superimposed concentration profiles of thin film and interdiffusion were analyzed for the simultaneous determination of tracer and interdiffusion coefficients. Processed concentration profiles obtained from the diffusion experiments were also fitted with simple Gaussian distribution function. Results were compared to existing literature data obtained independently by radiotracer experiments, and an excellent agreement has been observed.
\end{abstract}

Keywords experimental techniques · interdiffusion - tracer diffusivity

Yongho Sohn

Yongho.Sohn@ucf.edu

1 Department of Material Science and Engineering, Advanced Materials Processing and Analysis Center, University of Central Florida, Orlando, FL 32816

2 Centre for Mass and Thermal Transport in Engineering Materials, University of Newcastle, Callaghan, NSW 2308, Australia

\section{Introduction}

Diffusion coefficients are important material property parameters that help the understanding of phase transformations and microstructural development. For metallic alloys, investigation of diffusion typically involves measurement of interdiffusion coefficients, intrinsic diffusion coefficients and/or tracer diffusion coefficients. Both interdiffusion and intrinsic diffusion coefficients are 'thermo-kinetic' parameters since they include both thermodynamic and kinetic terms based on the Darken model. Tracer diffusion experiments provide the most fundamental information about the number of jumps (per unit time) an atom makes between the lattice sites. ${ }^{[1]}$ The tracer diffusion coefficient is a kinetic parameter that is typically employed for constructing mobility diffusion databases, and can be coupled with thermodynamic information to constitute interdiffusion and intrinsic diffusion coefficients.

Traditional techniques ${ }^{[2,3]}$ for experimentally determining the tracer diffusion coefficient in alloys can be challenging and costly due to the use of isotopes and the number of experiments required to assess composition and temperature dependence. Therefore, an efficient way of obtaining tracer diffusion coefficients is highly sought after. Furthermore, simultaneous measurement of tracer and interdiffusion coefficients would provide an efficient and consistent way to distinguish the kinetic (e.g., mobility) and thermodynamic (e.g., thermodynamic factor, $\Phi$ ) influences on the overall diffusion process in metallic alloys, for example, through the Darken model.

A new formalism ${ }^{[4,5]}$ for simultaneous measurement of isotope tracers and interdiffusion coefficients in multicomponent alloys has been recently reported. This formalism utilized Onsager's phenomenological relationships ${ }^{[6]}$ combined with the Boltzmann-Matano 
transformation. In this study, we implement an analytical method based on this new formalism to simultaneously determine interdiffusion coefficients, $\tilde{D}$ and tracer $\left(D_{\mathrm{Cu}}^{*}\right)$ diffusion coefficients in the $\mathrm{Cu}-\mathrm{Ni}$ system at 800, 900 and $1000{ }^{\circ} \mathrm{C}$. The $\mathrm{Cu}-\mathrm{Ni}$ system was chosen for this investigation to demonstrate the validity of the new analytical framework, because there are ample data on both tracer diffusion and interdiffusion in the literature.

\section{Analytical Framework}

Consider an isothermal annealing of a binary diffusion couple that consists of alloys with compositions $A_{x} B_{(1-\mathrm{x})}$ $-A_{y} B_{(1-y)}$. Within the couple, consider that atoms of the same type $(A)$ have fraction of them that is situated at a given (known) location in the couple and has a different relative composition from that at the remaining locations. In other words, the $A B$ couple constructed in such a way that there is a fraction of $A$ atoms that can be separated from the rest of the $A$ atoms based on its location. Therefore, it is possible to distinguish them and $A$ atoms can be split into two groups: atoms $A 1$ and $A 2$. Then, due to the difference in their specific locations and compositions, the respective thermodynamic forces $\left(X_{A 1}\right.$ and $\left.X_{A 2}\right)$ of these atoms will not be equal. Furthermore, the flux of $A$ atoms, $J_{A}$, can be split into two fluxes, $J_{A 1}$ and $J_{A 2}$ accordingly. Given this information, the Onsager expressions for the diffusion fluxes of the atomic components $A$ and $B$ in the lattice coordinate system can be written as:

$J_{A 1}=L_{A 1 A 1} X_{A 1}+L_{A 1 A 2} X_{A 2}+L_{A 1 B} X_{B}$

$J_{A 2}=L_{A 1 A 2} X_{A 1}+L_{A 2 A 2} X_{A 2}+L_{A 2 B} X_{B}$

$J_{B}=L_{A 1 B} X_{A 1}+L_{A 2 B} X_{A 2}+L_{B B} X_{B}$

where the $L_{i j}$ 's are the Onsager phenomenological transport coefficients, the $X_{j}$ 's are the forces acting on atoms, $J_{A}=$ $J_{A 1}+J_{A 2}$ and $X_{A 1} \neq X_{A 2}$.

In general, in an isothermal, isobaric system with a composition gradient, the driving force $X_{A}$ can be expressed in terms of the chemical potential gradient:

$X_{A}=-\nabla \mu_{A}=-k T\left(\frac{\nabla c_{A}}{c_{A}}+\nabla \ln \left(\gamma_{A}\right)\right)$

where $\gamma_{A}$ is the activity coefficient of the $A$ component, and for simplicity only a unidirectional flow in the $x$ direction is considered. The two types of $A$ atoms constitute the total composition of $A$ atoms, $c_{A}$ :

$c_{A 1}+c_{A 2}=c_{A}$

For a system in mechanical equilibrium, there is a restriction on the forces (also known as the Gibbs-Duhem relation):
$c_{A 1} X_{A 1}+c_{A 2} X_{A 2}+c_{B} X_{B}=0$

Since in a diffusion couple experiment, fluxes are typically measured in the laboratory coordinate system $(\tilde{J})$, transformation between the lattice and laboratory coordinate system is necessary. Kirkwood et al. ${ }^{[7]}$ showed that the fluxes with respect to any two reference frames are related. Using this relationship, fluxes in the laboratory coordinates reference frame can be written as:

$\tilde{J}_{i}=J_{i}+v N c_{i}, \quad i=A, A 1, A 2, B$,

where $v$ is the lattice drift velocity, $N$ is the number of lattice sites per unit volume, $\tilde{J}_{i}$ is the flux of type $i$ atoms in the laboratory coordinate system and $J_{i}$ is the flux of type $i$ atoms in the lattice coordinate system. Then

$v N=\left(\tilde{J}_{A}-J_{A}\right) / c_{A}$

and

$\tilde{J}_{A 1}=J_{A 1}-\frac{c_{A 1}}{c_{A}} J_{A}+\frac{c_{A 1}}{c_{A}} \tilde{J}_{A}$

and similarly, for $\tilde{J}_{A 2}$ :

$\tilde{J}_{A 2}=J_{A 2}-\frac{c_{A 2}}{c_{A}} J_{A}+\frac{c_{A 2}}{c_{A}} \tilde{J}_{A}$.

In $\mathrm{Eq} 7$, there are two terms still in the lattice reference frame, which need to be further replaced with experimentally measurable terms.

After Darken's initial approximate relations that were first published, ${ }^{[8]}$ Manning $^{[9]}$ presented more accurate relations between the tracer diffusion coefficients and the Onsager transport coefficients using a random alloy model. In this model, vacancies and different atoms are assumed to be distributed randomly in an alloy. More recently, relations between the Onsager transport coefficients and tracer diffusion coefficients were derived without the dependency on the atomic model. ${ }^{[10]}$ Utilization of these relationships yields the following expressions:

$$
\begin{aligned}
& L_{A 1 A 1}=\frac{N c_{A 1}}{k T}\left(D_{A}^{*}+c_{A 1} F_{\mathrm{A}}\right) \\
& L_{A 2 A 2}=\frac{N c_{A 2}}{k T}\left(D_{A}^{*}+c_{A 2} F_{\mathrm{A}}\right) \\
& L_{B B}=\frac{N c_{B}}{k T}\left(D_{B}^{*}+c_{B} F_{\mathrm{B}}\right) \\
& L_{A 1 A 2}=\frac{N c_{A 1} c_{A 2}}{k T} F_{A} \\
& L_{A 1 B}=\frac{N c_{A 1} c_{B}}{k T} F_{A B} \\
& L_{A 2 B}=\frac{N c_{A 2} c_{B}}{k T} F_{A B}
\end{aligned}
$$


where $D_{A}^{*}$ and $D_{B}^{*}$ are the tracer diffusion coefficients, $F_{A}$ is a correlation function between the movements of two different $A$ atoms, $F_{B}$ is a correlation function between the movements of two different $B$ atoms, and $F_{A B}$ is a crosscorrelation function between the movements of $A$ and $B$ atoms.

The fluxes in the lattice coordinate system, Eq 1, together with expressions for the Onsager transport coefficients Eq 9, can now be substituted into Eq 7 to yield the following expressions:

$\tilde{J}_{A 1}=\frac{N}{k T} D_{A}^{*} c_{A 1}\left(X_{A 1}-X_{A}\right)+\frac{c_{A 1}}{c_{A}} \tilde{J}_{A}$

Making use of Eq 2 and 10 can be rewritten as:

$\tilde{J}_{A 1}=-N D_{A}^{*}\left(\nabla c_{A 1}-\frac{c_{A 1}}{c_{A}} \nabla c_{A}\right)+\frac{c_{A 1}}{c_{A}} \tilde{J}_{A}$

Similarly:

$\tilde{J}_{A 2}=-N D_{A}^{*}\left(\nabla c_{A 2}-\frac{c_{A 2}}{c_{A}} \nabla c_{A}\right)+\frac{c_{A 2}}{c_{A}} \tilde{J}_{A}$

In Eq 11, all the terms can be defined based on the laboratory reference frame once the concentration profiles are extracted from the experimental data.

The equations described above can be used to extract the tracer diffusion coefficient $D_{A}^{*}$ with a known interdiffusion concentration profile of $A$ that includes the partial concentration profile of $A 1$. Now, assuming the diffusion couple was annealed for a time $t$, and $N$ (the number of lattice sites per unit volume) is not changing with composition, the diffusion equations for components $A, A 1$ and $B$ can be written as:

$\frac{\partial c_{A}}{\partial t}=-\frac{\nabla \tilde{J}_{A}}{N}=\nabla\left(\tilde{D} \nabla c_{A}\right)$

$\frac{\partial c_{A 1}}{\partial t}=\nabla\left[D_{A}^{*} \nabla c_{A 1}+\left(D_{A}^{*}-\tilde{D}\right) \frac{c_{A 1}}{c_{A}} \nabla c_{B}\right]$

$\frac{\partial c_{B}}{\partial t}=\nabla\left(\tilde{D} \nabla c_{B}\right)$

where $\tilde{D}$ is the (standard) interdiffusion coefficient. When the Boltzmann variable transform, $\lambda=x / \sqrt{t}$ is applied to Eq 13 with the assumption that

$c_{A 1}=\frac{f(\lambda)}{\sqrt{t}}$

and using the transformations of the partial derivatives with respect to $x$ and $t$ into the ordinary derivative with respect to $\lambda$ :

$\frac{\partial}{\partial x}=\frac{1}{\sqrt{t}} \frac{\mathrm{d}}{\mathrm{d} \lambda}$ $\frac{\partial}{\partial t}=\frac{\lambda}{2 t} \frac{\mathrm{d}}{\mathrm{d} \lambda}$

the following relation can be obtained:

$-\left(f+\lambda \frac{\mathrm{d} f}{\mathrm{~d} \lambda}\right)=2 \frac{\mathrm{d}}{\mathrm{d} \lambda}\left\{D_{A}^{*} \frac{\mathrm{d} f}{\mathrm{~d} \lambda}+\left(D_{A}^{*}-\tilde{D}\right) \frac{f}{c_{A}} \frac{\mathrm{d} c_{B}}{\mathrm{~d} \lambda}\right\}$,

or

$-\frac{\mathrm{d}(\lambda f)}{\mathrm{d} \lambda}=2 \frac{\mathrm{d}}{\mathrm{d} \lambda}\left\{D_{A}^{*} \frac{\mathrm{d} f}{\mathrm{~d} \lambda}+\left(D_{A}^{*}-\tilde{D}\right) \frac{f}{c_{A}} \frac{\mathrm{d} c_{B}}{\mathrm{~d} \lambda}\right\}$,

where $f, \tilde{D}$ and $D_{A}^{*}$ are approximated as functions of $\lambda$ only. After integrating $\mathrm{Eq} 19$ with respect to the variable $\lambda$ we have that:

$-\frac{\lambda f}{2}=D_{A}^{*} \frac{\mathrm{d} f}{\mathrm{~d} \lambda}+\left(D_{A}^{*}-\tilde{D}\right) \frac{f}{c_{A}} \frac{\mathrm{d} c_{B}}{\mathrm{~d} \lambda}$

Using

$\frac{\mathrm{d} c_{B}}{\mathrm{~d} \lambda}=-\frac{\mathrm{d} c_{A}}{\mathrm{~d} \lambda}$

and dividing Eq 20 by $f$ yields:

$-\frac{\lambda}{2}=D_{A}^{*} \frac{\mathrm{d} \ln \left(c_{A 1}\right)}{\mathrm{d} \lambda}-\left(D_{A}^{*}-\tilde{D}\right) \frac{\mathrm{d} \ln \left(c_{A}\right)}{\mathrm{d} \lambda}$

Since the anneal time, $t$, will always be a constant, Eq 22 can be rewritten as:

$-\frac{x}{2 t}=D_{A}^{*} \frac{\mathrm{d} \ln \left(c_{A 1}\right)}{\mathrm{d} x}-\left(D_{A}^{*}-\tilde{D}\right) \frac{\mathrm{d} \ln \left(c_{A}\right)}{\mathrm{d} x}$

After isolating the tracer diffusion coefficient on the lefthand-side, and rearranging the remaining terms, the following expressions can be obtained:

$D_{A}^{*}=-\left(\frac{x}{2 t}+\tilde{D} \frac{\mathrm{d} \ln \left(c_{A}\right)}{\mathrm{d} x}\right) /\left(\frac{\mathrm{d} \ln \left(c_{A 1}\right)}{\mathrm{d} x}-\frac{\mathrm{d} \ln \left(c_{A}\right)}{\mathrm{d} x}\right)$

or,

$D_{A}^{*}=-\left(\frac{x c_{A 1}}{2 t}+\tilde{D} \frac{c_{A 1}}{c_{A}} \frac{\mathrm{d} c_{A}}{\mathrm{~d} x}\right) /\left(\frac{\mathrm{d} c_{A 1}}{\mathrm{~d} x}-\frac{c_{A 1}}{c_{A}} \frac{\mathrm{d} c_{A}}{\mathrm{~d} x}\right)$

In the standard Boltzmann-Matano analysis, the location of the Matano plane must be determined. Similarly, in the present analysis, the origin of the $\lambda$ axis must be determined. Since Eq 21 does not include any integral terms, the origin of the $\lambda$ can be taken as $\lambda+a^{*}$, where $a^{*}$ is a constant representing the shift of the position of the $\lambda$. Then $\lambda$ in Eq 23 can be simply replaced by the $\lambda+a^{*}$ term:

$-\frac{\lambda+a^{*}}{2}=D_{A}^{*} \frac{\mathrm{d} \ln \left(c_{A 1}\right)}{\mathrm{d} \lambda}-\left(D_{A}^{*}-\tilde{D}\right) \frac{\mathrm{d} \ln \left(c_{A}\right)}{\mathrm{d} \lambda}$

For the solution of $\mathrm{Eq} 26, a=a^{*} \sqrt{t}$ relationship can be 
used where $a$ is also a constant. The role of this constant in the equation is to control the sign of the numerator and denominator. Thus, determining the value of $a$ should be done in a way that the resulting tracer coefficient will be positive. Finally, the most convenient form of the equation can be written as:

$D_{A}^{*}=-\left(\frac{(x+a)}{2 t}+\tilde{D} \frac{\mathrm{d} \ln c_{A 2}}{\mathrm{~d} x}\right) /\left(\frac{\mathrm{d} \ln \left(c_{A 1} / c_{A 2}\right)}{\mathrm{d} x}\right)$

\section{Experimental Procedure}

In this study, the $\mathrm{Cu}-\mathrm{Ni}$ system was selected for experiments because a large number of diffusion data are available in the literature. Three $\mathrm{Cu}-\mathrm{Ni}$ alloys with varying compositions (Cu-25at.\%Ni, Cu-50at.\%Ni, Cu-75at.\%Ni) and pure $\mathrm{Ni}$ discs were used for diffusion couple preparations. The alloys in the diffusion couple were arranged for simultaneous measurement of the interdiffusion and isotopic diffusion profiles. Figure 1 shows a typical arrangement of diffusion couple assembly. A thin film of "isotopic" $\mathrm{Cu}$ was placed between 'Metal 2' and 'Metal 3', while 'Metal 1' and 'Metal 2' were in contact with one another without the 'isotopic' thin film. Thus, only interdiffusion takes place between 'Metal 1' and 'Metal 2', while the thin-film $\mathrm{Cu}$ 'isotopic' diffusion occurs in addition to the interdiffusion between 'Metal 2' and 'Metal 3', as schematically illustrated in Fig. 1. A list of all diffusion couples assembled, annealed and analyzed in this study is given in Table 1. To further verify the repeatability and consistency of this study, diffusion couple experiments, 2 , 4, 7 and 9 were repeated. These couples were denoted, for example, 2A and 2B for diffusion couple experiment 2.

For diffusion couple assembly, alloy surfaces were ground and metallographically polished down to a $1 \mu \mathrm{m}$ surface finish. Samples were then ultrasonically cleaned with high purity ethanol. On selected alloys, electron beam physical vapor deposition (EB-PVD) technique was employed for $\mathrm{Cu}$ deposition. $\mathrm{Cu}$ deposition was carried out with the main chamber pressure of $1.2 \times 10^{-7}$ Torr, power of $10 \mathrm{kV}$, current of $320 \mathrm{~mA}$, and a deposition rate of $1.0 \sim 1.4 \AA / \mathrm{s}$. Deposition rate was monitored by using an oscillating crystal. Based on the target thickness of $3 \mu \mathrm{m}$, the $\mathrm{Cu}$ deposition period lasted 80 minutes. The prepared alloys were assembled into diffusion couples as listed in Table 1. They were held together by a stainless steel jig with alumina $\left(\mathrm{Al}_{2} \mathrm{O}_{3}\right)$ spacers to avoid any reaction between the stainless steel and the diffusion couple. The

Fig. 1 A schematic diagram of diffusion couples and corresponding concentration profiles employed for simultaneous measurement of interdiffusion and tracer diffusion coefficients
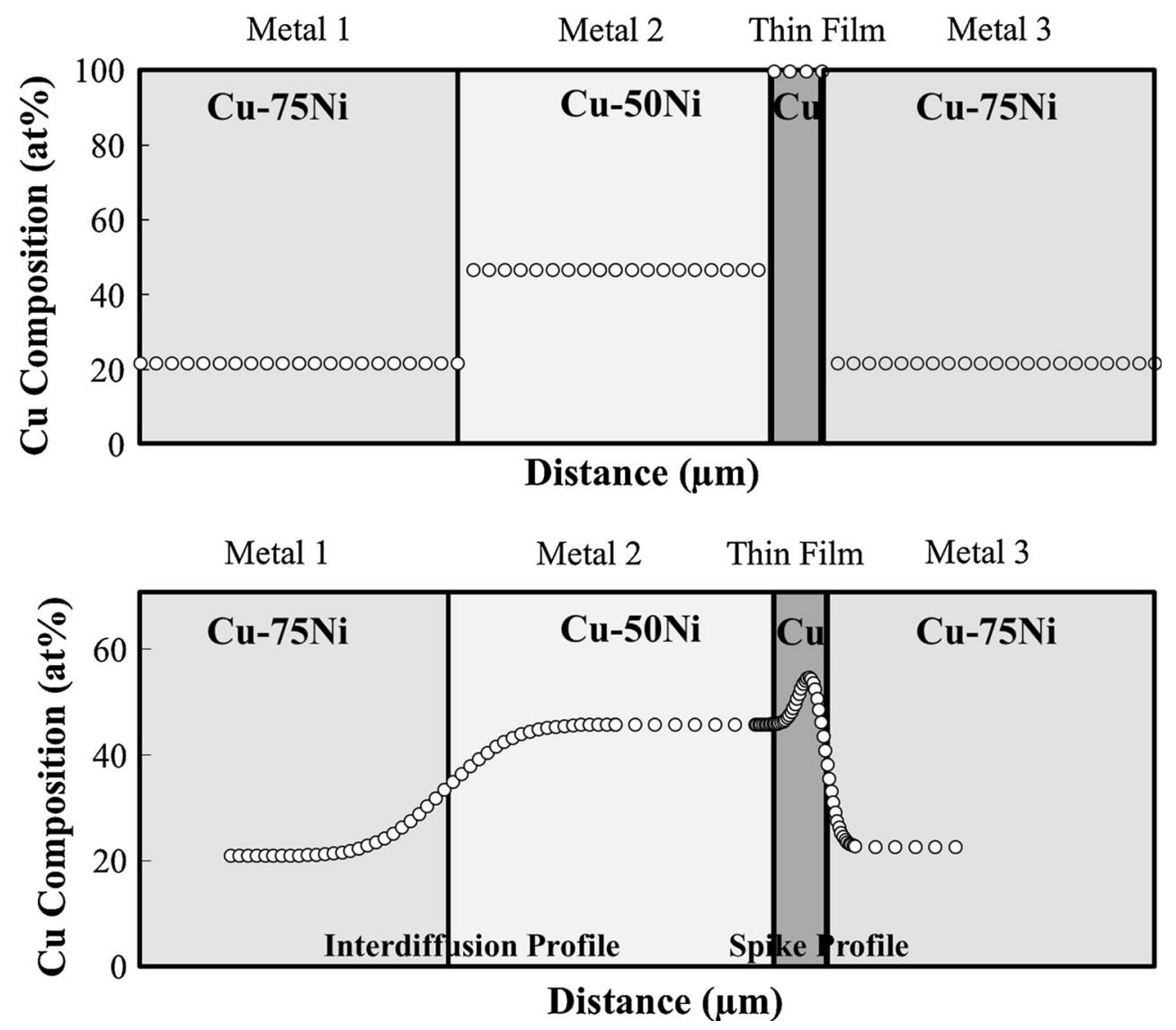
Table 1 Stacking sequence, anneal temperatures and anneal times of diffusion couples

\begin{tabular}{|c|c|c|c|c|c|c|}
\hline \multirow[t]{2}{*}{ Couple } & \multicolumn{4}{|c|}{ Stacking sequence } & \multirow[t]{2}{*}{ Temp., ${ }^{\circ} \mathrm{C}$} & \multirow[t]{2}{*}{ Time, } \\
\hline & Metal 1 & Metal 2 & Thin film & Metal 3 & & \\
\hline 1 & $\mathrm{Cu} 50 \mathrm{Ni}$ & $\mathrm{Cu} 25 \mathrm{Ni}$ & $\mathrm{Cu}$ & Cu50Ni & 1000 & 900 \\
\hline 2 & $\mathrm{Cu} 50 \mathrm{Ni}$ & $\mathrm{Cu} 25 \mathrm{Ni}$ & $\mathrm{Cu}$ & $\mathrm{Cu} 50 \mathrm{Ni}$ & 900 & 900 \\
\hline 3 & $\mathrm{Cu} 50 \mathrm{Ni}$ & $\mathrm{Cu} 25 \mathrm{Ni}$ & $\mathrm{Cu}$ & $\mathrm{Cu} 50 \mathrm{Ni}$ & 800 & 5400 \\
\hline 4 & $\mathrm{Cu} 75 \mathrm{Ni}$ & $\mathrm{Cu} 50 \mathrm{Ni}$ & $\mathrm{Cu}$ & $\mathrm{Cu} 75 \mathrm{Ni}$ & 1000 & 7200 \\
\hline 5 & $\mathrm{Cu} 75 \mathrm{Ni}$ & $\mathrm{Cu} 50 \mathrm{Ni}$ & $\mathrm{Cu}$ & $\mathrm{Cu} 75 \mathrm{Ni}$ & 900 & 18,000 \\
\hline 6 & $\mathrm{Cu} 75 \mathrm{Ni}$ & $\mathrm{Cu} 50 \mathrm{Ni}$ & $\mathrm{Cu}$ & $\mathrm{Cu} 75 \mathrm{Ni}$ & 800 & 75,600 \\
\hline 7 & $\mathrm{Cu} 75 \mathrm{Ni}$ & $\mathrm{Ni}$ & $\mathrm{Cu}$ & $\mathrm{Cu} 75 \mathrm{Ni}$ & 1000 & 3600 \\
\hline 8 & $\mathrm{Cu} 75 \mathrm{Ni}$ & $\mathrm{Ni}$ & $\mathrm{Cu}$ & $\mathrm{Cu} 75 \mathrm{Ni}$ & 900 & 3600 \\
\hline 9 & $\mathrm{Cu} 75 \mathrm{Ni}$ & $\mathrm{Ni}$ & $\mathrm{Cu}$ & $\mathrm{Cu} 75 \mathrm{Ni}$ & 800 & 10,800 \\
\hline
\end{tabular}

assembled diffusion couples were encapsulated in quartz tubes using an oxy-propylene torch. Prior to sealing, the capsules were repeatedly evacuated and flushed with $\mathrm{Ar}$ and $\mathrm{H}_{2}$. Quartz tubes were sealed when a vacuum of $8 \times 10^{-6}$ Torr or better was achieved.

The annealing of the diffusion couples was performed with a Lindberg/Blue ${ }^{\mathrm{TM}}$ three-zone tube furnace. In Table 1 the time and annealing temperature for all diffusion couples are given. After annealing, the quartz capsules were carefully removed from the furnace and quenched by breaking the capsules in cold water. After quenching, the diffusion couples were mounted in epoxy, cross-sectioned and metallographically polished down to $1 \mu \mathrm{m}$ for characterization. The interdiffusion zone was examined by a Zeiss ${ }^{\mathrm{TM}}$ Ultra-55 field emission scanning electron microscopy (FE-SEM) equipped with x-ray energy dispersive spectroscopy (XEDS). Standardless quantification via XEDS was employed to obtain concentration profiles across both diffusion zones: one with interdiffusion only and the other with the imposed thin film (e.g., tracer and interdiffusion). Concentration profiles extracted from XEDS data were iteratively fitted using OriginPro ${ }^{\mathrm{TM}} 8.5$ software using the expression: ${ }^{[11]}$

$c(x)=\frac{p_{1}+p_{3} x+p_{5} x^{2}+p_{7} x^{3}}{1+p_{2} x+p_{4} x^{2}+p_{6} x^{3}}$

The goodness of the fit was determined by reduced chisquare and the adjusted $R^{2}$ values approached 1 .

\section{Interdiffusion}

In this study, the Boltzmann-Matano method was adopted for the determination of interdiffusion coefficients as a function of composition using the expression:
$\tilde{D}_{i}=\frac{\frac{1}{2 t} \int_{C_{i}^{ \pm \infty}}^{C_{i}}\left(x-x_{o}\right) \mathrm{d} C_{i}}{\frac{\partial C_{i}}{\partial x}}$

Heumann and Grundhoff ${ }^{[12]}$ and Iijima et al. ${ }^{[13]}$ determined the interdiffusion coefficients in the $\mathrm{Cu}-\mathrm{Ni}$ system at $1000{ }^{\circ} \mathrm{C}$ for the entire composition range. Interdiffusion coefficients determined by Iijima et al. ${ }^{[13]}$ were slightly higher. Figure 2(a) shows the interdiffusion coefficients determined in this study as a function of $\mathrm{Cu}$ composition obtained from diffusion couples, \#1, \#4, and \#7 annealed at $1000{ }^{\circ} \mathrm{C}$ as listed in Table 1 . Magnitude and composition dependence of interdiffusion coefficients at $1000{ }^{\circ} \mathrm{C}$ were in good agreement with those reported by Heumann and Grundhoff. ${ }^{[12]}$

Trønsdal and Sørum ${ }^{[14]}$ quantitatively determined the temperature and concentration dependence of interdiffusion coefficients at medium concentrations for the temperature range $700-1000{ }^{\circ} \mathrm{C}$. The interdiffusion coefficients reported by Trønsdal and $\operatorname{Sørum}^{[14]}$ at 900 and $800{ }^{\circ} \mathrm{C}$ were significantly higher than the interdiffusion coefficients determined by this study as presented in Fig. 2(b) and (c). Hayashi et al. ${ }^{[15]}$ also examined the $\mathrm{Cu}-$ $\mathrm{Ni}$ interdiffusion for the temperature range 765 and $906{ }^{\circ} \mathrm{C}$. While the composition-dependence was similar, the magnitude of the interdiffusion coefficients was lower than those determined in this study. Impurity diffusion coefficients reported by Askil, ${ }^{[16]}$ also plotted in Fig. 2(b) demonstrate that the magnitude and the trend in composition-dependence are more consistent with interdiffusion coefficients determined in this study.

Zhao et al. ${ }^{[17]}$ investigated interdiffusion coefficient of $\mathrm{Cu}$ and $\mathrm{Ni}$ in the temperature range of 650 and $850{ }^{\circ} \mathrm{C}$ as presented in Fig. 2(c). Interdiffusion coefficients at $800{ }^{\circ} \mathrm{C}$ for selected compositions were also reported by Trønsdal and Sørum ${ }^{[14]}$ as represented in Fig. 2(c). Results obtained from this study demonstrate that the composition-dependence is consistent among all studies but the magnitude 

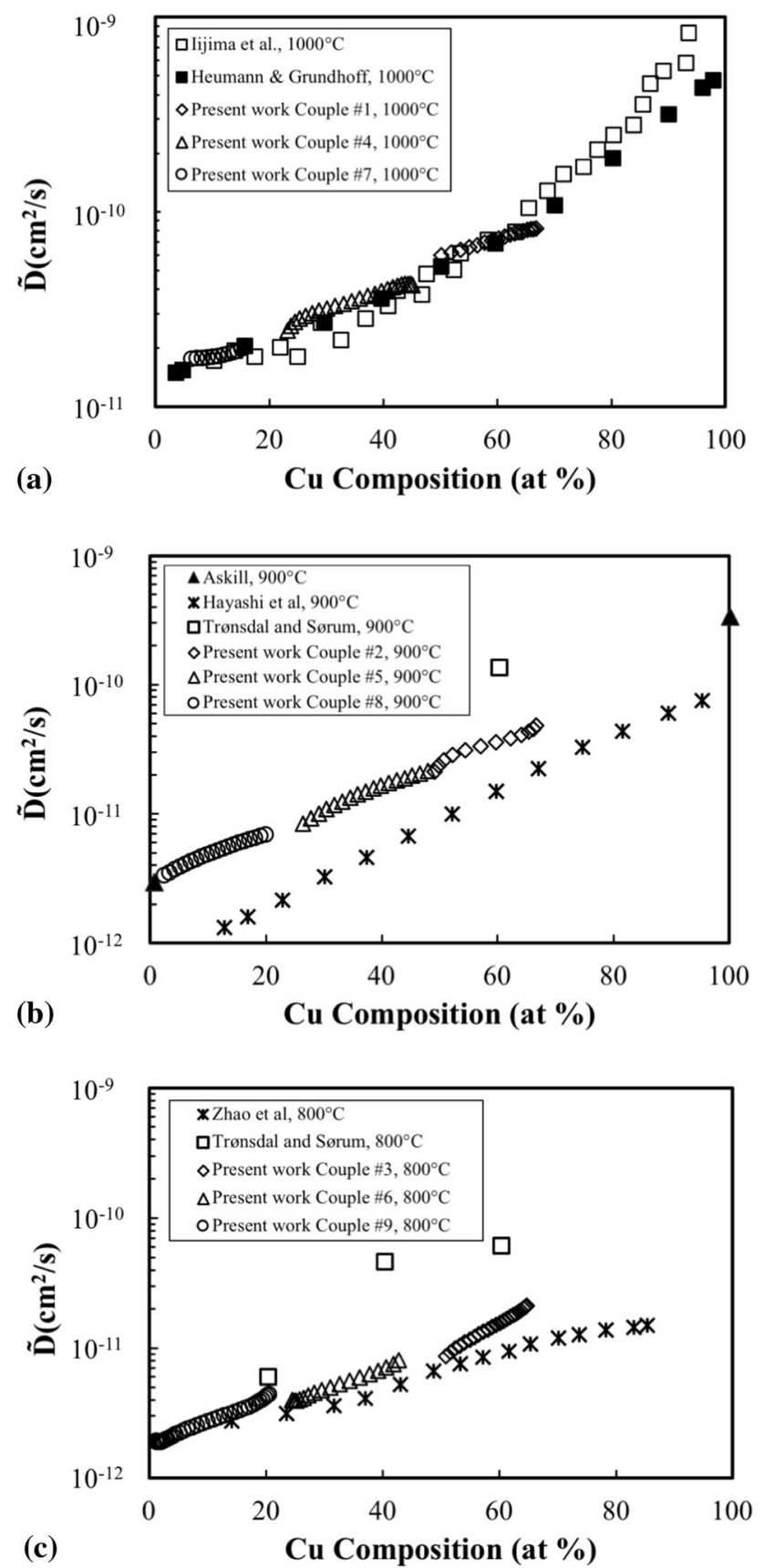

Fig. 2 Interdiffusion coefficients determined as a function of composition at (a) 1000 , (b) 900 , (c) $800{ }^{\circ} \mathrm{C}$

determined by this study falls between those determined by Zhao et al. ${ }^{[17]}$ and Trønsdal and Sørum. ${ }^{[14]}$

\section{Tracer Diffusion Coefficient Determination from Thin Film Diffusion}

As was explained above, the diffusion couples assembly consisted of a part with just interdiffusion and the other part with a thin film sandwiched in-between as shown in
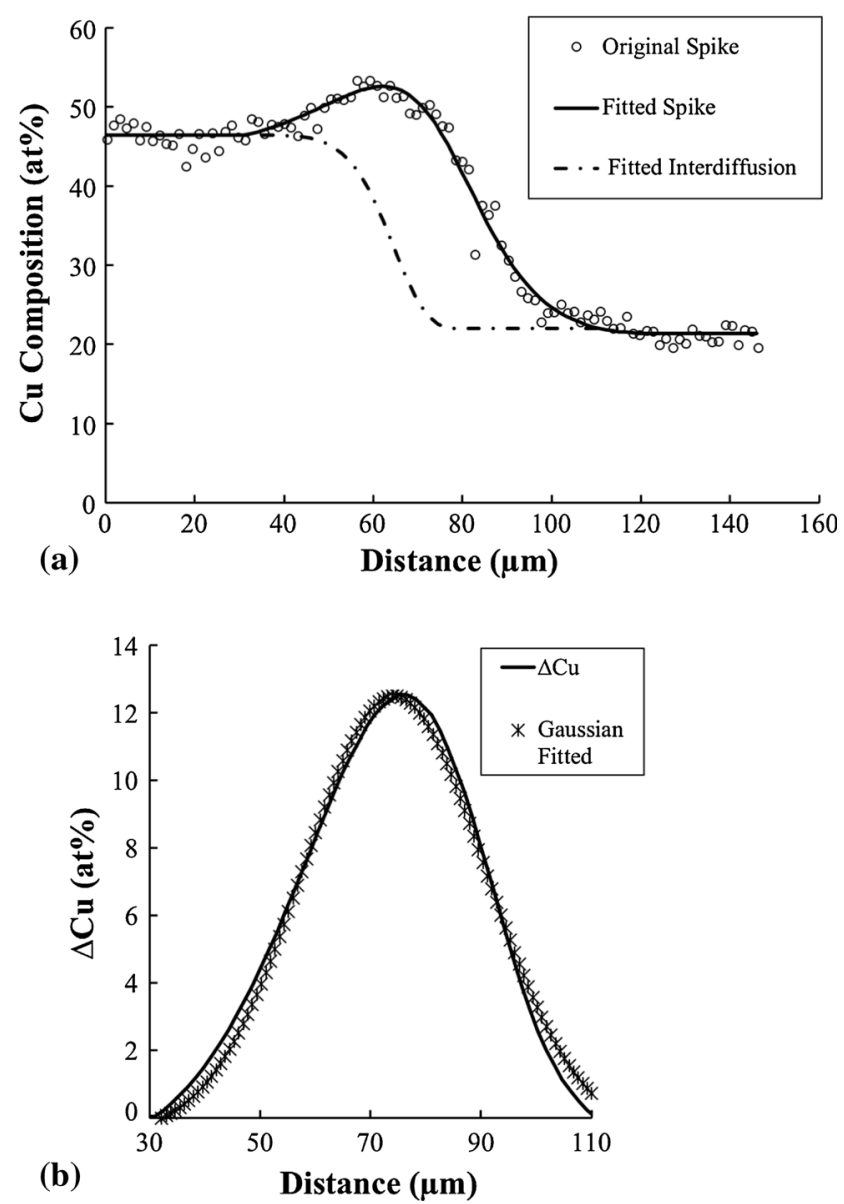

Fig. 3 (a) Concentration profile that includes both interdiffusion and thin film diffusion and (b) concentration profile from thin film diffusion extracted $\left(\Delta \mathrm{Cu}=\mathrm{Cu}-\mathrm{Cu}_{2}\right)$ and Gaussian distribution function fitting

Fig. 1. Annealing of the couple created a local 'spike' in the composition of one of the alloy components as schematically illustrated in Fig. 1 (i.e., pure $\mathrm{Cu}$ thin film), which include contributions from both, interdiffusion and thin film diffusion. The analysis of the thin film diffusion profile (spike) gave the total composition of $\mathrm{Cu}$ and then the analysis of the standard interdiffusion profile allow for separation of the fraction (i.e., $\mathrm{c}_{\mathrm{Cu} 2}$ fraction) of total $\mathrm{Cu}$ atoms (Eq 3 with $\mathrm{A}=\mathrm{Cu}$ ) and corresponding atomic fluxes as expressed by Eq 11a and 11b. This approach of distinguishing thin film and interdiffusion profiles, i.e., $\mathrm{Cu}$ and $\mathrm{Cu}_{2}$ profiles, is based on the assumption that the thickness of pure $\mathrm{Cu}$ layer is negligibly small.

In order to obtain the $\mathrm{c}_{\mathrm{Cu} 1}$ profile, the interdiffusion contribution is subtracted from the total $\mathrm{c}_{\mathrm{Cu}}$ profile. This was achieved by taking the mirror image of the interdiffusion profile and subtracting $c_{\mathrm{Cu}_{2}}$ profile from total $c_{\mathrm{Cu}}$ profile as demonstrated in Fig. 3. Once the concentration profiles were obtained, Eq 27 was used to determine the tracer diffusion coefficient. 
Tracer diffusion coefficient, $D_{\mathrm{Cu}}^{*}$ was determined based on the formalism developed by Belova et al. ${ }^{[4]}$ and is plotted as a function of $\mathrm{Cu}$ composition near 50 at.\% in Fig. 4. The application of the formalism successfully estimated the tracer diffusion coefficient of $\mathrm{Cu}$ however, it did not give a reliable compositional dependence. Therefore, the tracer diffusion coefficient, $D_{\mathrm{Cu}}^{*}$ was also calculated utilizing the Gaussian distribution function applied to the $\mathrm{c}_{\mathrm{Cu} 1}$ profile and plotted against the corresponding spike composition. Table 2 summarizes the tracer diffusion coefficient, $D_{\mathrm{Cu}}^{*}$ obtained by application of the Belova et al. formalism as a function of $\mathrm{Cu}$ composition (at.\%) and the tracer diffusion coefficient calculated using the Gaussian distribution function. When the existing literature tracer diffusion data obtained by radioactive isotopes studies ${ }^{[2]}$ compared with the data obtained by this study utilizing Gaussian distribution function, good agreement was found, as presented in Fig. 5.

To further verify the applicability and consistency of the methodology, diffusion couples with varying terminal alloy compositions $(\mathrm{Cu}, \mathrm{Cu}-25 \mathrm{Ni}, \mathrm{Cu}-50 \mathrm{Ni}, \mathrm{Cu}-75 \mathrm{Ni}, \mathrm{Ni})$ were annealed at 800,900 and $1000{ }^{\circ} \mathrm{C}$, and analyzed for the

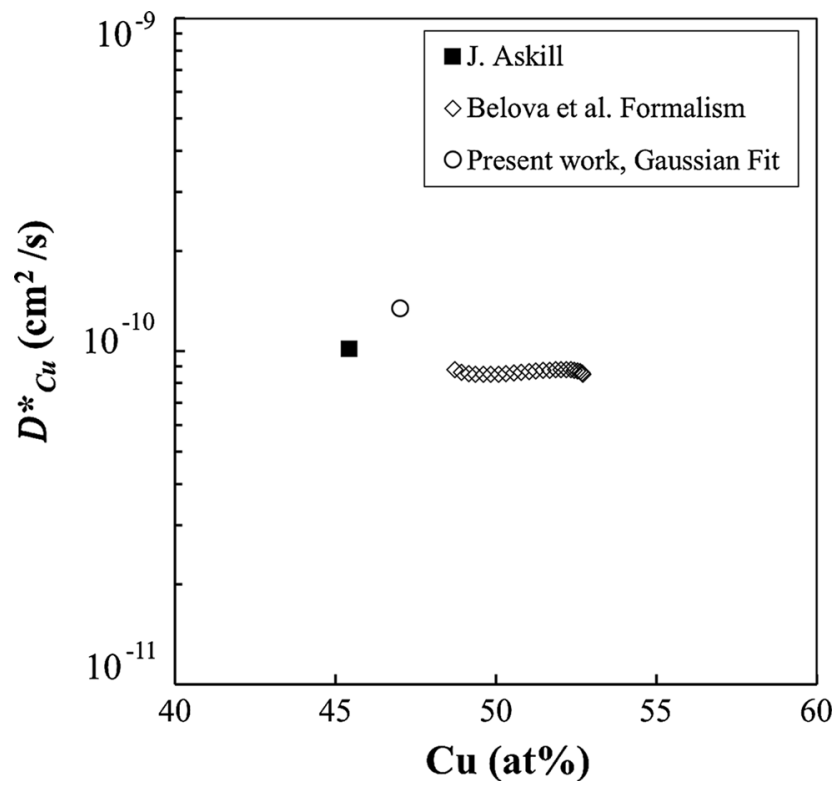

Fig. 4 Tracer diffusion coefficient, $D_{\mathrm{Cu}}^{*}$ determined by compositiondependent analysis and Gaussian distribution function, independent of composition

Table 2 Tracer diffusion coefficient derived from the Belova et al. formalism and Gaussian distribution function simultaneous determination of tracer and interdiffusion coefficients as a function of composition. In order to determine the standard deviation for each temperature range and to demonstrate the repeatability of the experiments, independent diffusion couple experiments were also carried out, and corresponding standard deviations were determined. Denotations of " $A$ " and "B" were used for the repeated couples. Table 3 summarizes the tracer diffusion coefficients, $D_{\mathrm{Cu}}^{*}$ obtained from each diffusion couple examined in this study. Results from 1000,900 and $800{ }^{\circ} \mathrm{C}$ were also compared to existing literature data ${ }^{[16,18-20]}$ obtained independently by radiotracer experiments as shown in Fig. 5. It can be concluded that, in this investigation, the measurement of tracer diffusion coefficients in the $\mathrm{Cu}-\mathrm{Ni}$ interdiffusion experimental set-up making use of the novel analytical framework produced reliable results. This validates that the approach can be applied to other alloys as well to provide kinetic (i.e., tracer diffusion coefficients) and thermo-kinetic data (i.e., interdiffusion coefficients) from the same experiment without the use of radioactive or stable isotopes.

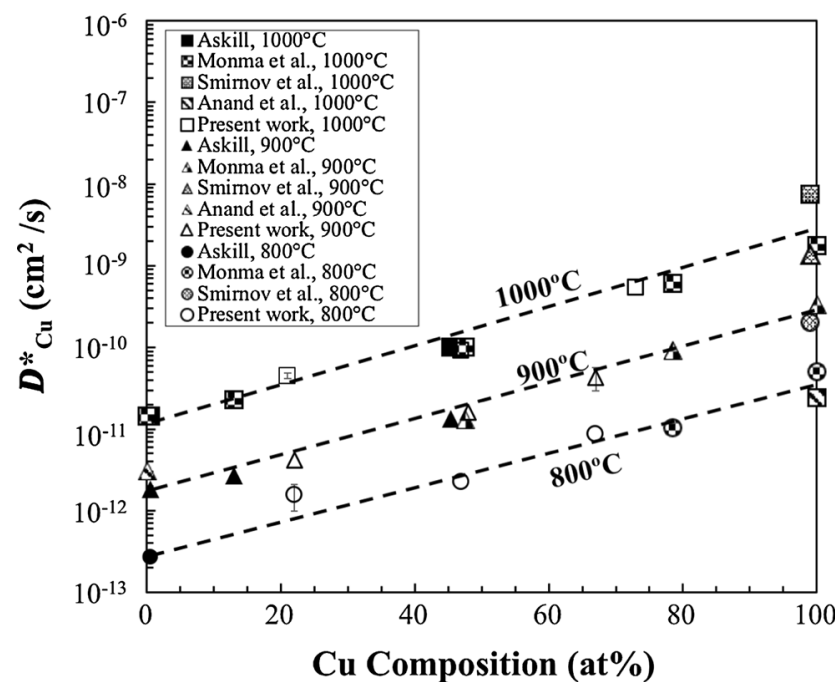

Fig. 5 Tracer diffusion coefficient, $D_{\mathrm{Cu}}^{*}$ determined as a function of composition based on Gaussian distribution function fitting of thin film diffusion

\begin{tabular}{lcc}
\hline $\mathrm{Cu}$, at. $\%$ & $\begin{array}{c}D_{\mathrm{Cu}}^{*} \\
\text { Belova et al. formalism, } \mathrm{cm}^{2} / \mathrm{s}\end{array}$ & $\begin{array}{c}D_{\mathrm{Cu}}^{*} \\
\text { Gaussian distribution function, } \mathrm{cm}^{2} / \mathrm{s}\end{array}$ \\
\hline 47 & $8.6 \times 10^{-11}$ & $1.3 \times 10^{-10}$ \\
48 & $8.6 \times 10^{-11}$ & \\
49 & $8.6 \times 10^{-11}$ & \\
50 & $8.6 \times 10^{-11}$ & \\
\hline
\end{tabular}


Table 3 Summary of tracer diffusion coefficients obtained from each couple annealed

\begin{tabular}{|c|c|c|c|c|c|c|}
\hline Couple & $\mathrm{Cu}$ composition, at. $\%$ & Temp., ${ }^{\circ} \mathrm{C}$ & Time, s & $D_{\mathrm{Cu}}^{*}, \mathrm{~cm}^{2} / \mathrm{s}$ & Average $D_{\mathrm{Cu}}^{*}, \mathrm{~cm}^{2} / \mathrm{s}$ & Standard deviation \\
\hline 1 & 73 & 1000 & 900 & $5.4 \times 10^{-10}$ & $\ldots$ & $\ldots$ \\
\hline $2-\mathrm{A}$ & 67 & 900 & 900 & $5.7 \times 10^{-11}$ & $4.3 \times 10^{-11}$ & $7 \times 10^{-12}$ \\
\hline $2-\mathrm{B}$ & 67 & 900 & 900 & $4.6 \times 10^{-11}$ & & \\
\hline 3 & 67 & 800 & 5400 & $8.0 \times 10^{-12}$ & $\ldots$ & $\ldots$ \\
\hline 4-A & 47 & 1000 & 7200 & $1.8 \times 10^{-10}$ & $1.4 \times 10^{-10}$ & $6 \times 10^{-11}$ \\
\hline 4-B & 47 & 1000 & 7200 & $9.0 \times 10^{-11}$ & & \\
\hline 5 & 48 & 900 & 18,000 & $1.6 \times 10^{-11}$ & $\ldots$ & $\ldots$ \\
\hline 6 & 47 & 800 & 75,600 & $2.5 \times 10^{-12}$ & $\ldots$ & $\ldots$ \\
\hline 7-A & 21 & 1000 & 3600 & $4.1 \times 10^{-11}$ & $4.5 \times 10^{-11}$ & $4 \times 10^{-12}$ \\
\hline 7-B & 21 & 1000 & 3600 & $4.9 \times 10^{-11}$ & & \\
\hline 8 & 22 & 900 & 3600 & $4.2 \times 10^{-12}$ & $\ldots$ & $\ldots$ \\
\hline 9-A & 22 & 800 & 10,800 & $9.9 \times 10^{-13}$ & $8.7 \times 10^{-13}$ & $2 \times 10^{-13}$ \\
\hline 9-B & 22 & 800 & 10,800 & $7.5 \times 10^{-13}$ & & \\
\hline
\end{tabular}

\section{Conclusions}

The tracer diffusion coefficient, $D_{\mathrm{Cu}}^{*}$ and interdiffusion coefficients have been simultaneously determined using the experimental methodology based on the new formalism and Gaussian distribution function fitting. This was performed using standard diffusion couple experiments without any radioactive or stable isotopes. Results produced were in excellent agreement with previously reported values determined independently by radiotracer and interdiffusion experiments, when Gaussian solution of thin-film concentration profile was utilized. This investigation demonstrated that the new formalism can be successfully applied to binary systems without using radiotracers and self/tracer diffusivities can be obtained from traditional diffusion couple experiments.

Acknowledgments IVB and GEM would like to acknowledge support of the Australian Research Council through its Discovery Project Grants Scheme (DP170101812).

\section{References}

1. H. Mehrer, Diffusion in Solids: Fundamentals, Methods, Materials, Diffusion-Controlled Processes, Springer, Berlin, 2007, p 212-214

2. A. Benninghoven, F.G. Rudenauer, and H.W. Werner, Secondary Ion Mass Spectrometry: Basic Concepts, Instrumental Aspects, Applications and Trends, John Wiley \& Sons, New York, 1987, p $257-258$

3. A. Paul, T. Laurila, V. Vuorinen, and S.V. Divinski, Thermodynamics, Diffusion \& The Kirkendall Effect in Solids, Springer, Berlin, 2014, p 167

4. I.V. Belova, Y.H. Sohn, and G.E. Murch, Measurement of Tracer Diffusion Coefficients in an Interdiffusion Context for Multicomponent Alloys, Philos. Mag. Lett., 2015, 95(8), p 416-424

5. I. Belova, N.S. Kulkarni, Y.H. Sohn, and G. Murch, Simultaneous Measurement of Tracer and Interdiffusion Coefficients: An
Isotopic Phenomenological Diffusion Formalism for the Binary Alloy, Philos. Mag., 2013, 93(26), p 3515-3526

6. L. Onsager, Reciprocal Relations in Irreversible Processes, I, Phys. Rev., 1931, 37(4), p 405

7. J.G. Kirkwood, R.L. Baldwin, P.J. Dunlop, L.J. Gosting, and G. Kegeles, Flow Equations and Frames of Reference for Isothermal Diffusion in Liquids, J. Chem. Phys., 1960, 33(5), p 1505

8. L.S. Darken, Diffusion, Mobility and Their Interrelation Through Free Energy in Binary Metallic Systems, Trans. AIME, 1948, 175, p 184-194

9. J.R. Manning, Correlation Factors for Diffusion in Nondilute Alloys, Phys. Rev. B, 1971, 4(4), p 1111-1121

10. A.R. Allnatt and A.B. Lidiard, Atomic Transport in Solids, Cambridge University Press, Cambridge, 1993, p 161-180

11. D. Liu, L. Zhang, Y. Du, H. Xu, and Z. Jin, Ternary Diffusion in Cu-Rich fcc Cu-Al-Si Alloys at 1073 K, J. Alloys. Compd., 2013, 566, p 156-163

12. T. Heumann and K. Grundhoff, Diffusion and Kirkendall Effect in the Cu-Ni System, Z. Metallkunde, 1972, 63(4), p 173-180, in German

13. Y. Iijima, K. Hirano, and M. Kikuchi, Determination of Intrinsic Diffusion Coefficients in a Wide Concentration Range of a Cu-Ni Couple by the Multiple Markers Method, Trans. Jpn. Inst. Metals, 1982, 23(1), p 19-23

14. G.O. Trønsdal and H. Sørum, Interdiffusion in $\mathrm{Cu}-\mathrm{Ni}, \mathrm{Co}-\mathrm{Ni}$, and Co-Cu, Phys. Status Solidi (B), 1964, 4(3), p 493-498

15. E. Hayashi, Y. Kurokawa, and Y. Fukai, Hydrogen-Induced Enhancement of Interdiffusion in Cu-Ni Diffusion Couples, Phys. Rev. Lett., 1998, 80(25), p 5588-5590

16. J. Askill, Tracer Diffusion Data for Metals, Alloys and Simple Oxides, Springer, Berlin, 1970, p 31-80

17. J. Zhao, J.E. Garay, U. Anselmi-Tamburini, and Z.A. Munir, Directional Electromigration Enhanced Interdiffususion in the Cu-Ni System, J. Appl. Phys., 2007, 102(11), p 114902

18. K. Monma, H. Suto, and H. Oikawa, Diffusion of Ni 63 and cu 64 in Nickel-Copper Alloys (On the Relation Between High Temperature Creep and Diffusion in Nickel Base Solid Solutions), $J$. Jpn. Inst. Metals, 1964, 28(188), p 192-196, in Japanese

19. E.A. Smirnov, L.I. Ivanov, and E.A. Abranyan, Experimental study of self-diffusion in copper, Izv. Akad. Nauk SSSR Metal., 1967, 168

20. M.S. Anand, S.P. Murarka, and R.P. Agarwala, Diffusion of Copper in Nickel and Aluminum, J. Appl. Phys., 1965, 36(12), p 3860-3862 